\title{
Cubicity of Interval Graphs and the Claw Number
}

\author{
Abhijin Adiga $^{\star}$ and L. Sunil Chandran ${ }^{\star \star}$
}

\begin{abstract}
Let $G(V, E)$ be a simple, undirected graph where $V$ is the set of vertices and $E$ is the set of edges. A $b$-dimensional cube is a Cartesian product $I_{1} \times I_{2} \times \cdots \times I_{b}$, where each $I_{i}$ is a closed interval of unit length on the real line. The cubicity of $G$, denoted by $\operatorname{cub}(G)$ is the minimum positive integer $b$ such that the vertices in $G$ can be mapped to axis parallel $b$-dimensional cubes in such a way that two vertices are adjacent in $G$ if and only if their assigned cubes intersect. An interval graph is a graph that can be represented as the intersection of intervals on the real line - i.e., the vertices of an interval graph can be mapped to intervals on the real line such that two vertices are adjacent if and only if their corresponding intervals overlap. Suppose $S(m)$ denotes a star graph on $m+1$ nodes. We define claw number $\psi(G)$ of the graph to be the largest positive integer $m$ such that $S(m)$ is an induced subgraph of $G$. It can be easily shown that the cubicity of any graph is at least $\left\lceil\log _{2} \psi(G)\right\rceil$.

In this paper, we show that, for an interval graph $G\left\lceil\log _{2} \psi(G)\right\rceil \leq \operatorname{cub}(G) \leq\left\lceil\log _{2} \psi(G)\right\rceil+2$. It is not clear whether the upper bound of $\left\lceil\log _{2} \psi(G)\right\rceil+2$ is tight: till now we are unable to find any interval graph with $\operatorname{cub}(G)>\left\lceil\log _{2} \psi(G)\right\rceil$. We also show that, for an interval graph $G$, $\operatorname{cub}(G) \leq\left\lceil\log _{2} \alpha\right\rceil$, where $\alpha$ is the independence number of $G$. Therefore, in the special case of $\psi(G)=\alpha, \operatorname{cub}(G)$ is exactly $\left\lceil\log _{2} \alpha\right\rceil$.

The concept of cubicity can be generalized by considering boxes instead of cubes. A $b$-dimensional box is a Cartesian product $I_{1} \times I_{2} \times \cdots \times I_{b}$, where each $I_{i}$ is a closed interval on the real line. The boxicity of a graph, denoted $\operatorname{box}(G)$, is the minimum $k$ such that $G$ is the intersection graph of $k$-dimensional boxes. It is clear that $\operatorname{box}(G) \leq \operatorname{cub}(G)$. From the above result, it follows that for any graph $G, \operatorname{cub}(G) \leq \operatorname{box}(G)\left\lceil\log _{2} \alpha\right\rceil$.
\end{abstract}

Keywords: Cubicity, boxicity, interval graphs, indifference graphs, claw number.

\section{Introduction}

Let $G(V, E)$ be a simple, undirected graph where $V$ is the set of vertices and $E$ is the set of edges. A $b$-dimensional box is a Cartesian product $R_{1} \times R_{2} \times \cdots \times R_{b}$, where each $R_{i}$ is a closed interval on the real line. When each interval has unit length, we will call such a box a $b$-dimensional cube. The cubicity (respectively boxicity) of $G$, denoted by $\operatorname{cub}(G)(\operatorname{box}(G))$, is the minimum positive integer $b$ such that the vertices in $G$ can be mapped to axis parallel $b$-dimensional cubes (boxes) in such a way that two vertices are adjacent in $G$ if and only if their assigned cubes (boxes) intersect. Cubicity and boxicity were introduced by Roberts in [20]. Yannakakis [24] proved that it is NP-complete to determine if the cubicity of a graph is at most 3. It was shown by Cozzens [8] that computing the boxicity of a graph is NP-hard. This was strengthened by Kratochvil [17] who showed that deciding whether boxicity of a graph is at most 2 itself is NP-complete.

Roberts [20] showed that for any graph $G, \operatorname{cub}(G) \leq\lfloor 2 n / 3\rfloor$ and $\operatorname{box}(G) \leq\lfloor n / 2\rfloor$. By definition, the cubicity and boxicity of a complete graph is 0 . The cube representation of special classes of graphs like hypercubes,

\footnotetext{
* Indian Institute of Science, Dept. of Computer Science and Automation, Bangalore 560012, India. email: abhijin@csa.iisc.ernet.in

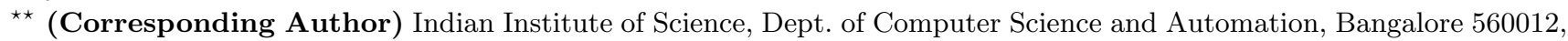
India. email: sunil@csa.iisc.ernet.in This work was supported by DST grant SR/S3/EECE/62/2006 and Infosys Fellowship.
} 
co-bipartite and complete multipartite graphs were investigated in $[7,24,20]$. Scheinerman [21] showed that the boxicity of outer planar graphs is at most 2. Thomassen [22] proved that the boxicity of planar graphs is at most 3. Adiga [1] showed that the cubicity of threshold graphs is exactly $\left\lceil\log _{2} \alpha\right\rceil$ where $\alpha$ is the independence number of the graph. In [9], Cozzens and Roberts studied the boxicity of split graphs. Boxicity and cubicity find applications in niche overlap and problems of fleet maintenance in operations research (for more information see [9]). If a graph has a box or cube representation in low dimension, some well known NP-hard problems become either polynomial time solvable or will have a much better approximation ratio. For example, it is easy to see that the maximum clique problem is polynomial-time solvable for graphs with bounded boxicity, while in general it is an NP-hard problem. Finding the maximum independent set is hard to approximate within a factor $n^{\frac{1}{2}-\epsilon}$ for general graphs. However, it is approximable to a factor of $\left\lfloor 1+\frac{1}{c} \log n\right\rfloor^{d-1}$ given a box representation in $d$ dimension. It is interesting to note that coloring problems on low boxicity graphs were considered as early as 1948 [2]. Kostochka [16] provides an extensive survey on colouring problems of intersection graphs. In [15, 11] the complexity of finding the maximum independent set in bounded boxicity graphs is considered. In $[14,10]$ cubicity has been studied in comparison with sphericity. Some other related references are $[4,6,23,19]$.

In this paper, we consider the cubicity of interval graphs. Graphs with boxicity at most 1 are precisely the well-studied class of interval graphs. A graph is an interval graph if and only if its vertices can be mapped to intervals on the real line such that two vertices are adjacent if and only if their corresponding intervals overlap. Similarly, a graph has cubicity at most 1 if and only if it is an indifference graph (also known as unit-interval graph or proper interval graph). We define indifference graph more formally later in the paper. It is easy to see that boxicity and cubicity are generalizations of the concept of interval graphs and indifference graphs respectively. From the definition of boxicity and cubicity, it is easy to see that any cube representation of a graph will also serve as a box representation. Hence, $\operatorname{box}(G) \leq \operatorname{cub}(G)$. Therefore, it is indeed interesting to ask the following question: what is the cubicity of a graph whose boxicity is 1 ?

Chandran and Mathew [5] showed that cubicity of an interval graph is at most $\left\lceil\log _{2}|V|\right\rceil$. They gave a constructive proof where all vertices were first numbered distinctly from $\{1, \ldots,|V|\}$ and the cube representing a vertex was determined by the binary representation of the number assigned to it. This was later improved to $\left\lceil\log _{2} \Delta\right\rceil+4$ in [3], where $\Delta$ is the maximum degree of $G$. In that paper, the vertices are ordered in a particular manner and then grouped into disjoint blocks of $2 \Delta$ vertices. We improve this bound further. To state our result, we first introduce a parameter called claw number of a graph. Recall that a star graph on $n$ vertices is the complete bipartite graph $K_{1, n-1}$. We denote it by $S(n-1)$.

Definition 1. The claw number $\psi(G)$ of a graph $G$ is the largest positive integer $m$ such that $S(m)$ is an induced subgraph of $G$.

Our result is as follows:

Theorem 1. Let $G$ be an interval graph with claw number $\psi$ and independence number $\alpha$.

$$
\left\lceil\log _{2} \psi\right\rceil \leq \operatorname{cub}(G) \leq \min \left(\left\lceil\log _{2} \psi\right\rceil+2,\left\lceil\log _{2} \alpha\right\rceil\right) .
$$

It is not clear whether the upper bound of $\left\lceil\log _{2} \psi\right\rceil+2$ is tight. We have not been able to find any interval graph with cubicity greater than $\left\lceil\log _{2} \psi\right\rceil$. Also, notice that $\alpha$ can be arbitrarily high compared to $\psi$. Consider for example, a path on $n>2$ vertices for which $\psi=2$ and $\alpha=\lceil n / 2\rceil$.

For the special case of $\psi=\alpha, \operatorname{cub}(G)$ is exactly $\left\lceil\log _{2} \alpha\right\rceil$. It is to be observed that if a graph has a universal vertex (i.e. a vertex adjacent to all other vertices), then, $\psi=\alpha$. For example, a connected threshold graph is an interval graph which has a universal vertex. Threshold graphs are an important and well-studied class of graphs 
[18]. It is clear that the cubicity of a connected threshold graph is exactly $\left\lceil\log _{2} \alpha\right\rceil$. This result was proved in [1] using a different constructive method. Theorem 1 is therefore a generalization of this result. To compare the result of this paper with the result $\operatorname{cub}(G) \leq \log _{2} \Delta+4$ given in [3] note that $\Delta$ can be arbitrarily high compared to $\psi$. One example is a complete graph, where $\psi=\alpha=1$, whereas $\Delta=|V|-1$.

Also, this particular upper bound of $\left\lceil\log _{2} \alpha\right\rceil$ allows us to bound the cubicity of any arbitrary graph in terms of its boxicity. It is stated below.

Corollary 1. Let $G$ be any graph. $\operatorname{cub}(G) \leq \operatorname{box}(G)\left\lceil\log _{2} \alpha\right\rceil$.

This is an improvement over the upperbound $\operatorname{cub}(G) \leq \operatorname{box}(G)\left\lceil\log _{2}|V|\right\rceil$ given by Chandran and Mathew [5].

The star graph $S(3)$ is also known as a claw. Claw free graphs are those which do not contain an induced copy of a claw. An interval graph is claw free if and only if it is an indifference graph [13]. Our result can be considered as an extension of this result by observing that for an indifference graph, cubicity is at most 1 and its claw number is at most 2.

\subsection{Preliminaries and Results}

In this section, we mention some useful properties and results regarding interval graphs and cubicity. Here, we give a formal definition of indifference graphs which we make use of in later sections.

Definition 2. Indifference graph: A graph $G(V, E)$ is an indifference graph if and only if there exists a function $\Pi: V \longrightarrow \mathbb{R}$ such that for two distinct vertices $u$ and $v, u$ and $v$ are adjacent if and only if $|\Pi(u)-\Pi(v)| \leq t$, for some fixed positive real number $t$.

It is easy to see that a graph has cubicity 1 if and only if it is an indifference graph.

Property 1. (See Golumbic [13] for a proof.) A graph $G$ is an interval graph if and only if its maximal cliques can be linearly ordered such that for every vertex $u$ the maximal cliques containing $u$ occur consecutively.

For a graph $G(V, E)$, let $G_{i}\left(V, E_{i}\right), i \in\{1,2, \ldots, k\}$ be such that $E=E_{1} \cap E_{2} \cap \cdots \cap E_{k}$. Then we say that $G$ is

the intersection of $G_{i}$ 's $1 \leq i \leq k$ and denote it as $G=\bigcap_{i=1}^{k} G_{i}$. Cubicity (Boxicity respectively) can be stated in terms of intersection of indifference graphs (interval graphs) as follows:

Lemma 1. Roberts [20] The cubicity (boxicity) of a graph $G$ is the minimum positive integer $b$ such that $G$ is the intersection of $b$ indifference graphs (interval graphs). Moreover, if $G=\bigcap_{i=0}^{m-1} G_{i}$, for some graphs $G_{i}$, then, $\operatorname{cub}(G) \leq \sum_{i=0}^{m-1} \operatorname{cub}\left(G_{i}\right)$ and $\operatorname{box}(G) \leq \sum_{i=0}^{m-1} \operatorname{box}\left(G_{i}\right)$.

The following result is easy to prove.

Lemma 2. Suppose $H$ is an induced subgraph of $G$, then $\operatorname{cub}(G) \geq \operatorname{cub}(H)$.

\section{Proof of Theorem 1}

The lower bound is easy to see and is as follows. Since the claw number of $G$ is $\psi$, it has an induced subgraph $S(\psi)$ and $\operatorname{cub}(S(\psi))=\left\lceil\log _{2} \psi\right\rceil$ (See Roberts [20]). By Lemma 2, $\operatorname{cub}(G) \geq \operatorname{cub}(S(\psi))=\left\lceil\log _{2} \psi\right\rceil$.

Our aim is to construct $\left\lceil\log _{2} \psi\right\rceil+2$ indifference graphs and show that $G$ is the intersection of these graphs, thereby proving the upper bound. First, we describe a vertex numbering which is essential for the construction of the indifference graphs. This vertex numbering is almost identical to that described by Gardi [12] where the author uses this numbering to partition interval graphs into proper interval subgraphs. 


\subsection{Vertex Labelling and the Primary Maximum Independent Set}

Let $G(V, E)$ be an interval graph. Let $\mathcal{C}: C_{0}, C_{1}, \ldots, C_{k-1}$ correspond to a linear ordering of maximal cliques satisfying Property 1 , where $C_{i}$ corresponds to the set of vertices in the $i$ th maximal clique. For a vertex $u$, let $c_{u}=\left\{i \mid u \in C_{i}\right\}$. It is clear that $c_{u}$ is a set of consecutive integers. Let $r(u)=\max _{i \in c_{u}} i$ and $l(u)=\min _{i \in c_{u}} i$ denote the rightmost and the leftmost cliques containing $u$ respectively. Note that two vertices $u$ and $v$ are adjacent if and only if $c_{u} \cap c_{v} \neq \varnothing$.

Let $\eta: V \longrightarrow \mathbb{Z}$ be a labelling of vertices obtained in the following manner: Choose a vertex $u_{0}$ such that $r\left(u_{0}\right) \leq r(v), \forall v \neq u_{0}$. Assign label 0 to $u_{0}$ and all vertices adjacent to $u_{0}$. $u_{0}$ will be considered as a special vertex among vertices labelled 0 . Continue the same way considering only the unlabelled vertices and numbering incrementally at each stage until all the vertices are labelled. Let $I_{\mathcal{C}}=\left\{u_{0}, u_{1}, \ldots\right\}$ be the collection of special vertices. We define the algorithm more formally below:

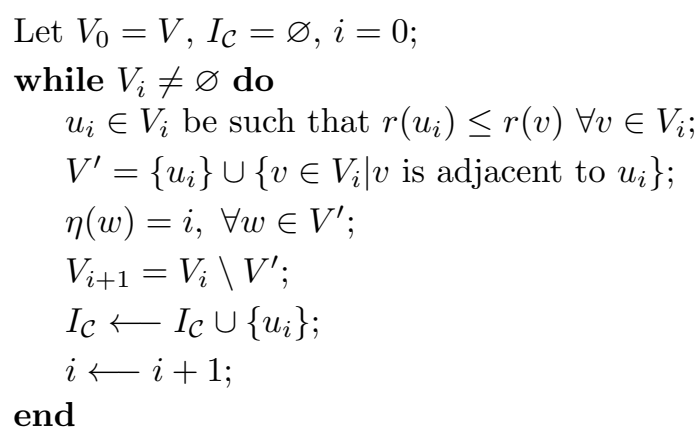

Observation 1. For any vertex $v, \eta(v) \leq i$ if and only if $l(v) \leq r\left(u_{i}\right)$.

Proof. Since $v$ is adjacent to $u_{\eta(v)}$, we have $l(v) \leq r\left(u_{\eta(v)}\right)$. It is clear that $r\left(u_{\eta(v)}\right) \leq r\left(u_{i}\right)$ since $\eta(v) \leq i$. Therefore, $l(v) \leq r\left(u_{i}\right)$.

Suppose $\eta(v)>i$. From the algorithm, it implies that $r(v)>r\left(u_{i}\right)$. Suppose $l(v) \leq r\left(u_{i}\right)$, that is $l(v) \leq r\left(u_{i}\right) \leq$ $r(v)$. This implies that $v$ is adjacent to $u_{i}$. Then, by the algorithm $\eta(v) \leq i$, a contradiction.

Observation 2. For two vertices $v$ and $w$, if $\eta(v)=\eta(w)$, then $v$ and $w$ are adjacent.

Proof. Let $\eta(v)=\eta(w)=i$. From Observation 1 and from the algorithm it follows that $l(v) \leq r\left(u_{i}\right) \leq r(v)$ and $l(w) \leq r\left(u_{i}\right) \leq r(w)$. Therefore, $r\left(u_{i}\right) \in c_{v} \cap c_{w}$. Hence proved.

Let $l$ be the number of iterations in the algorithm for graph $G$, i.e. $V_{l-1} \neq \varnothing$ and $V_{l}=\varnothing$.

Observation 3. $I_{\mathcal{C}}$ is a maximum independent set. Hence, $\left|I_{\mathcal{C}}\right|=\alpha$.

Proof. From the vertex numbering algorithm it is evident that $\mathcal{C}$ is an independent set. Suppose there exists an independent set of size greater than $l$. By pigeon hole principle, at least two vertices in this set will be assigned the same number and by Observation 2, they will be adjacent to each other, a contradiction.

$I_{\mathcal{C}}$ is crucial to our construction. From now on we refer to it as the primary independent set with respect to the linear ordering $\mathcal{C}$.

Observation 4. $0=r\left(u_{0}\right)<r\left(u_{1}\right)<\cdots<r\left(u_{\alpha-1}\right)=k-1$. 
Proof. From Observation 1 we see that for $i<\alpha-1, r\left(u_{i}\right)<l\left(u_{i+1}\right) \leq r\left(u_{i+1}\right)$. Hence, $r\left(u_{0}\right)<r\left(u_{1}\right)<\cdots<$ $r\left(u_{\alpha-1}\right)$. Next we show that $r\left(u_{0}\right)=0$ and $r\left(u_{\alpha-1}\right)=k-1$.

Suppose, $r\left(u_{0}\right) \neq 0$, then it is clear from the algorithm that for all vertices $v$ with $l(v)=0, r(v)>0$. This implies that $C_{0}$ is a subset of $C_{1}$, which contradicts the maximality of the cliques.

It is easy to see that $r\left(u_{\alpha-1}\right) \leq k-1$. Suppose $r\left(u_{\alpha-1}\right)=t<k-1$. Consider any vertex $v \in C_{k-1}$. Clearly, $r(v)=k-1>t$. Since $\eta(v) \leq \alpha-1$, from Observation $1, l(v) \leq t$. Therefore, $l(v) \leq t \leq r(v)$ which implies $v \in C_{t}$. Hence, $C_{k-1} \subseteq C_{t}$, which contradicts the maximality of the cliques.

\subsection{Defining the Indifference Graphs}

Recall that $\mathcal{C}: C_{0}, C_{1}, \ldots, C_{k-1}$ is a linear ordering of the maximal cliques of $G$ and $I_{\mathcal{C}}=\left\{u_{0}, \ldots, u_{\alpha-1}\right\}$ is the primary independent set with respect to $\mathcal{C}$. We can assume that $\psi(G)=2^{p}$, where $p$ is a positive integer. If not, we will work with another interval graph $G^{\prime}$ constructed in such a way that $\psi\left(G^{\prime}\right)=2^{p}$ and $G$ is an induced subgraph of $G^{\prime}$. To construct $G^{\prime}$ from $G$ we consider a vertex $v \in C_{k-1}$. Let $m$ be the largest positive integer such that there exists an induced $S(m)$ in $G$ with $v$ being the central vertex of this $S(m)$. To obtain $G^{\prime}$, we add $2^{p}-m$ new vertices $v_{0}, \ldots, v_{2^{p}-m-1}$ to $G$ such that they form an independent set and are adjacent to only $v$. Then it is easy to verify that $G^{\prime}$ would correspond to the following linear ordering of the maximal cliques: $\mathcal{C}^{\prime}: C_{0}^{\prime}, C_{1}^{\prime}, \ldots, C_{k+2^{p}-m-1}^{\prime}$, where, $C_{i}^{\prime}=C_{i} 0 \leq i \leq k-1$ and $C_{k+i}^{\prime}=\left\{v, v_{i}\right\} 0 \leq i \leq 2^{p}-m-1$. Clearly, $\mathcal{C}^{\prime}$ satisfies Property 1 and therefore $G^{\prime}$ is an interval graph. Moreover, we have an induced star $S\left(2^{p}\right)$ with $v$ as the central vertex. Clearly, the remaining vertices of $G$ are unaffected by this construction. Hence, $\psi\left(G^{\prime}\right)=2^{p}$.

Firstly, we provide an outline of the construction. We define a function $f(\cdot)$ which maps each maximal clique $C_{i}$ to a unique point on the real line. Then, we define a vertex labelling $\gamma(\cdot)$. In the ith indifference graph, for each vertex $v$, we consider the $i t h$ bit position of $\gamma(v)$ (more formally defined by the bit function $b_{i}(\cdot)$ ). If it is 0 , then, $v$ is assigned an interval of length $\psi-\frac{1}{2}$ whose right end point coincides with $f(r(v))$, i.e. the point on the real line representing the rightmost clique containing $v$. If it is 1 , then, $v$ is assigned an interval of length $\psi-\frac{1}{2}$ whose left end point coincides with $f(l(v))$, the point representing the leftmost clique containing $v$. The function $f:\{0, \ldots, k-1\} \longrightarrow \mathbb{R}$ is defined as follows:

1. $f\left(r\left(u_{0}\right)\right)=f(0)=0$.

2. For $j \in\left\{r\left(u_{i}\right)+1, \ldots, r\left(u_{i+1}\right)\right\}, f(j)=i+\frac{1}{2}+\frac{j-r\left(u_{i}\right)}{2\left(r\left(u_{i+1}\right)-r\left(u_{i}\right)\right)}$, for $0 \leq i<\alpha-1$.

Remark 1. From Observation 4 it is clear that $f$ is defined for each $i \in\{0,1, \ldots, k-1\}$. Moreover, $f$ is a strictly increasing function.

Given non-negative integers $a$ and $i$, the $i$ th bit function $b_{i}(\cdot)$ is defined as $b_{i}(a)=\left\lfloor\frac{a}{2^{i}}\right\rfloor \bmod 2$. Now we define $\gamma: V \longrightarrow\{\psi, \ldots, 3 \psi-1\}$ as follows:

$$
\gamma(u)=\left\{\begin{array}{l}
\eta(u) \bmod \psi+\psi, \text { if }\left\lfloor\frac{\eta(u)}{\psi}\right\rfloor \text { is even, } \\
\eta(u) \bmod \psi+2 \psi, \text { if }\left\lfloor\frac{\eta(u)}{\psi}\right\rfloor \text { is odd. }
\end{array}\right.
$$

Recall that $p=\log _{2} \psi$. Note that $\gamma(u)$ is defined in such a way that for $0 \leq i \leq p-1, b_{i}(\gamma(u))=b_{i}(\eta(u))$, i.e. the first $p$ bit positions of $\gamma(u)$ and $\eta(u)$ are identical. $b_{p}(\gamma(u))=1$ and $b_{p+1}(\gamma(u))=0$ when $\left\lfloor\frac{\eta(u)}{\psi}\right\rfloor$ is even and $b_{p}(\gamma(u))=0$ and $b_{p+1}(\gamma(u))=1$ when $\left\lfloor\frac{\eta(u)}{\psi}\right\rfloor$ is odd.

Now, we define $p+2=\log _{2} \psi+2$ indifference graphs $U_{0}, U_{1}, \ldots, U_{p+1}$ as follows. For each $U_{i}$ we define $\Pi_{i}: V \longrightarrow \mathbb{R}$ as per Definition 2: For $u \in V$,

$$
\Pi_{i}(u)= \begin{cases}f(r(u))-\psi+\frac{1}{2}, & \text { if } b_{i}(\gamma(u))=0, \\ f(l(u)), & \text { if } b_{i}(\gamma(u))=1,\end{cases}
$$


where $0 \leq i \leq p+1$. In the graph $U_{i}$, two vertices $u$ and $v$ are made adjacent if and only if $\left|\Pi_{i}(v)-\Pi_{i}(u)\right| \leq \psi-\frac{1}{2}$.

2.3 Proof of $G=\bigcap_{i=0}^{p+1} U_{i}$

Lemma 3. For any vertex $v, j \in c_{v}$ implies $f(j) \in\left[\Pi_{i}(v), \Pi_{i}(v)+\psi-\frac{1}{2}\right], 0 \leq i \leq p+1$.

Proof. Let $\eta(v)=m$. In order to handle some boundary cases, we define certain notations. If $q<0$, then, let $r\left(u_{q}\right)=-1$. If $q>\alpha-1$, then, let $r\left(u_{q}\right)=r\left(u_{\alpha-1}\right)=k-1$.

Claim 1. $j \in c_{v}$ implies $r\left(u_{m-1}\right)+1 \leq j \leq r\left(u_{m+\psi-1}\right)$.

Proof. If $m=0$, then it is clear that $l(v)=0=r\left(u_{0}\right)=r\left(u_{-1}\right)+1$. Suppose $m>0$. From Observation 1 it immediately follows that $l(v) \geq r\left(u_{m-1}\right)+1$ and therefore $j>r\left(u_{m-1}\right)$.

Next, we show that $j \leq r\left(u_{m+\psi-1}\right)$. Suppose $m \geq \alpha-\psi$. Since $q=m+\psi-1 \geq \alpha-1$, we have $r\left(u_{m+\psi-1}\right)=$ $r\left(u_{q}\right)=r\left(u_{\alpha-1}\right)=k-1$. But trivially, $j \leq k-1$. Hence, we assume that $m<\alpha-\psi$. Suppose $v=u_{m}$, then this is trivially true from Observation 4. Hence, we assume that $v \neq u_{m}$. Now, if there exists $j \in c_{v}$ such that $j>r\left(u_{m+\psi-1}\right)$, then $t=r\left(u_{m+\psi-1}\right)+1 \in c_{v}$, since by Observation $1, l(v) \leq r\left(u_{m+\psi-1}\right)$ and $c_{v}$ is a set of consecutive integers. There exists a vertex $w \in C_{t}$ such that $w \notin C_{q}$, for $q<t$, since otherwise $C_{t}$ will be a subset of $C_{t-1}$, contradicting the fact that $C_{i}$ s are maximal. Clearly $w \neq v$. Now we claim that $\eta(w)=m+\psi$. Since $l(w)=t>r\left(u_{m+\psi-1}\right)$, by Observation $1, \eta(w) \geq m+\psi$. Also $l\left(u_{m+\psi}\right)>r\left(u_{m+\psi-1}\right)$ which implies $r\left(u_{m+\psi}\right) \geq l\left(u_{m+\psi}\right) \geq t=l(w)$. By the algorithm to compute $\eta(\cdot), r(w) \geq r\left(u_{m+\psi}\right)$. Therefore, we have $l(w) \leq r\left(u_{m+\psi}\right) \leq r(w)$ which implies that $w$ is adjacent to $u_{m+\psi}$, which in turn means $\eta(w)=m+\psi$. Since $v, w \in C_{t}$, they are adjacent. Clearly, the vertex set $V^{\prime}=\left\{u_{m}, u_{m+1}, \ldots, u_{m+\psi-1}, w\right\}$ forms an independent set since $l(w)=t>r\left(u_{m+\psi-1}\right)$. Also, all the vertices of $V^{\prime}$ are adjacent to $v$ since, $l(v) \leq r\left(u_{m}\right) \leq r\left(u_{m+\psi-1}\right)<$ $l(w) \leq r(v)$. Therefore, $\{v\} \cup V^{\prime}$ forms an induced star $S(\psi+1)$, a contradiction. Hence, $j \leq r\left(u_{m+\psi-1}\right)$.

Claim 2. $f(r(v))-f(l(v))<\psi-\frac{1}{2}$.

Proof. From Claim 1 we have $r\left(u_{m-1}\right)+1 \leq l(v) \leq r(v) \leq r\left(u_{m+\psi-1}\right)$. Now, by the definition of $f$ and noting that $f$ is a strictly increasing function: $\max \left(m-\frac{1}{2}, 0\right)<f(l(v)) \leq f(r(v)) \leq \min (m+\psi-1, \alpha-1)$.

To complete the proof, we need to show that $[f(l(v)), f(r(v))] \subseteq\left[\Pi_{i}(v), \Pi_{i}(v)+\psi-\frac{1}{2}\right]$. If $b_{i}(\gamma(v))=0$,

$$
\left[\Pi_{i}(v), \Pi_{i}(v)+\psi-\frac{1}{2}\right]=\left[f(r(v))-\psi+\frac{1}{2}, f(r(v))\right]
$$

and if $b_{i}(\gamma(v))=1$,

$$
\left[\Pi_{i}(v), \Pi_{i}(v)+\psi-\frac{1}{2}\right]=\left[f(l(v)), f(l(v))+\psi-\frac{1}{2}\right] .
$$

In both cases it is sufficient to show that $f(l(v))>f(r(v))-\psi+\frac{1}{2}$, which immediately follows from Claim 2 .

Lemma 4. If $v, w \in V$ such that $v$ and $w$ are adjacent in $G$, then, $v$ and $w$ are adjacent in all the $p+2$ indifference graphs.

Proof. Since $v$ and $w$ are adjacent, $c_{v} \cap c_{w} \neq \varnothing$. From Lemma 3 it follows that if $j \in c_{v} \cap c_{w}$, then, $f(j) \in$ $\left[\Pi_{i}(v), \Pi_{i}(v)+\psi-\frac{1}{2}\right] \cap\left[\Pi_{i}(w), \Pi_{i}(w)+\psi-\frac{1}{2}\right]$ and hence, $\left|\Pi_{i}(v)-\Pi_{i}(w)\right| \leq \psi-\frac{1}{2}$ for $0 \leq i \leq p+1$.

Lemma 5. If $v, w \in V$ such that $v$ and $w$ are not adjacent in $G$, then there exists an indifference graph $U_{i}$, $i \in\{0, \ldots, p+1\}$, in which $u$ and $w$ are not adjacent. 
Proof. Without loss of generality we assume that $r(v)<l(w)$. Since $l(w)>r(v) \geq r\left(u_{\eta(v)}\right)$, from Observation 1 it follows that $\eta(v)<\eta(w)$.

Let $q_{v}=\left\lfloor\frac{\eta(v)}{\psi}\right\rfloor$ and $q_{w}=\left\lfloor\frac{\eta(w)}{\psi}\right\rfloor$. Now we consider the following cases separately:

1. Suppose $q_{w}=q_{v}$ : Recalling that $\eta(u) \bmod \psi=\gamma(u) \bmod \psi, \forall u, \gamma(v) \bmod \psi<\gamma(w) \bmod \psi$. This in turn implies that there exists $i<\log _{2} \psi=p$ such that $b_{i}(\gamma(v))=0$ and $b_{i}(\gamma(w))=1$. Then,

$$
\Pi_{i}(w)-\Pi_{i}(v)=f(l(w))-f(r(v))+\psi-\frac{1}{2}>\psi-\frac{1}{2} .
$$

The last inequality follows from the fact that, by definition $f(\cdot)$ is a strictly increasing function.

2. Suppose $q_{w}=q_{v}+1$ : If $q_{v}$ is odd, then $b_{p}(\gamma(v))=0$ and $b_{p}(\gamma(w))=1$ and therefore, as in Case $1, \Pi_{p}(w)-$ $\Pi_{p}(v)>\psi-\frac{1}{2}$. If $q_{v}$ is even, then $b_{p+1}(\gamma(v))=0$ and $b_{p+1}(\gamma(w))=1$ and similarly, $\Pi_{p+1}(w)-\Pi_{p+1}(v)>\psi-\frac{1}{2}$.

3. Suppose $q_{w}=q_{v}+2$ : If $q_{v}$ is even, then, $b_{p}(\gamma(v))=b_{p}(\gamma(w))=1$. $\Pi_{p}(w)-\Pi_{p}(v)=f(l(w))-f(l(v))$. For improved readability, let $c=q_{v} \psi$ and $d=q_{w} \psi$. Note that $\eta(w) \geq d$, and therefore, from Observation 1, $l(w) \geq r\left(u_{d-1}\right)+1$. Similarly, $\eta(v) \leq c+\psi-1$, and again from Observation $1, l(v) \leq r\left(u_{c+\psi-1}\right)$. Therefore,

$$
\begin{aligned}
f(l(w))-f(l(v)) & \geq f\left(r\left(u_{d-1}\right)+1\right)-f\left(r\left(u_{c+\psi-1}\right)\right) \\
& >\left(d-1+\frac{1}{2}\right)-(c+\psi-1) \\
& =\left(c+2 \psi+\frac{1}{2}\right)-(c+\psi) \\
& =\psi+\frac{1}{2}>\psi-\frac{1}{2} .
\end{aligned}
$$

If $q_{v}$ is odd, then, $b_{p+1}(\gamma(v))=b_{p+1}(\gamma(w))=1$ and in a similar manner as above, we can show that $\Pi_{p+1}(w)-\Pi_{p+1}(v)>\psi-\frac{1}{2}$.

4. Suppose $q_{w}>q_{v}+2$ : If $b_{p}(\gamma(v))=b_{p}(\gamma(w))=1$, then, we can show that $\Pi_{p}(w)-\Pi_{p}(v)>\psi-\frac{1}{2}$ in the same way as Case 3 . In a similar way, if $b_{p+1}(\gamma(v))=b_{p+1}(\gamma(w))=1$, we can show that $\Pi_{p+1}(w)-\Pi_{p+1}(v)>\psi-\frac{1}{2}$. Otherwise, from the definition of $\gamma(\cdot)$, it is easy to see that either $(1) b_{p}(\gamma(v))=0$ and $b_{p}(\gamma(w))=1$ OR (2) $b_{p+1}(\gamma(v))=0$ and $b_{p+1}(\gamma(w))=1$. As in Case 1 we can show that $\Pi_{p}(w)-\Pi_{p}(v)>\psi-\frac{1}{2}$ for $(1)$ and $\Pi_{p+1}(w)-\Pi_{p+1}(v)>\psi-\frac{1}{2}$ for $(2)$.

Hence proved.

Combining Lemmas 4 and 5, we have $G=\bigcap_{i=0}^{p+1} U_{i}$. Hence, we have proved Theorem 1.

Note that when $\psi=\alpha$, the independence number of $G$, we have $\left\lfloor\frac{\eta_{v}}{\psi}\right\rfloor=0$ and therefore $b_{p}(\gamma(v))=1$ and $b_{p+1}(\gamma(v))=0$ for all vertices $v \in V$. From this, it is easy to see that $U_{p}$ and $U_{p+1}$ will correspond to complete graphs. Therefore, cubicity of $G$ will be exactly $\left\lceil\log _{2} \alpha\right\rceil$.

Next we observe that, given any interval graph $G$, we can construct a graph $G^{\prime}$ by adding a universal vertex to $G$. It is easy to that $G^{\prime}$ is an interval graph which contains $G$ as an induced subgraph. Also, $\psi\left(G^{\prime}\right)=\alpha\left(G^{\prime}\right)=\alpha$. By Lemma 2, it follows that $\operatorname{cub}(G) \leq \operatorname{cub}\left(G^{\prime}\right)=\left\lceil\log _{2} \alpha\right\rceil$. Hence, we have proved that $\left\lceil\log _{2} \psi\right\rceil \leq \operatorname{cub}(G) \leq$ $\min \left(\left\lceil\log _{2} \psi\right\rceil+2,\left\lceil\log _{2} \alpha\right\rceil\right)$.

\subsection{Proof of Corollary 1}

Let $b=\operatorname{box}(G)$. By Lemma 1 , there exist $b$ interval graphs, say $G_{i}, 0 \leq i<b$, such that $G=\bigcap_{i=0}^{b-1} G_{i}$. Since each $G_{i}$ is a supergraph of $G, \alpha\left(G_{i}\right) \leq \alpha$. Therefore, $\operatorname{cub}\left(G_{i}\right) \leq\left\lceil\log _{2} \alpha\right\rceil$. Again by Lemma 1, we have $\operatorname{cub}(G) \leq \sum_{i=0}^{b-1} \operatorname{cub}\left(G_{i}\right) \leq \operatorname{box}(G)\left\lceil\log _{2} \alpha\right\rceil$. Hence, we have proved that $\operatorname{cub}(G) \leq \operatorname{box}(G)\left\lceil\log _{2} \alpha\right\rceil$. 
We observe that this inequality is tight. In fact, given any two positive integers $k$ and $l$, there exists a graph $G$ with $\operatorname{box}(G)=k, \alpha=l$ such that $\operatorname{cub}(G)=k\left\lceil\log _{2} l\right\rceil$. One such example is the complete $k$-partite graph with $|V|=k l$ (See Roberts [20] for proofs).

\section{References}

1. A. Adiga, Cubicity of threshold graphs, Disc. Math. 309 (8) (2009) 2535-2537.

2. A. Bielecki, Problem 56, Colloq. Math 1 (1948) 333.

3. L. S. Chandran, M. C. Francis, N. Sivadasan, On the cubicity of interval graphs, Graphs and Combinatorics 25 (2009) 169-179.

4. L. S. Chandran, M. C. Francis, N. Sivadasan, Boxicity and maximum degree, J. Combin. Theory Ser. B 98 (2) (2008) $443-445$.

5. L. S. Chandran, K. A. Mathew, An upper bound for cubicity in terms of boxicity, Disc. Math. (2008) doi:10.1016/j.disc.2008.04.011.

6. L. S. Chandran, N. Sivadasan, Boxicity and treewidth, J. Combin. Theory Ser. B 97 (5) (2007) 733-744.

7. L. S. Chandran, N. Sivadasan, The cubicity of hypercube graphs, Disc. Math. 308 (23) (2008) 5795-5800.

8. M. B. Cozzens, Higher and multi-dimensional analogues of interval graphs, Ph.D. thesis, Department of Mathematics, Rutgers University, New Brunswick, NJ (1981).

9. M. B. Cozzens, F. S. Roberts, Computing the boxicity of a graph by covering its complement by cointerval graphs, Disc. Appl. Math. 6 (1983) 217-228.

10. P. C. Fishburn, On the sphericity and cubicity of graphs, J. Combin. Theory Ser. B 35 (1983) 309-308.

11. R. J. Fowler, M. S. Paterson, S. L. Tanimoto, Optimal packing and covering in the plane are NP-complete, Information Processing letters 12 (3) (1981) 133-137.

12. F. Gardi, On partitioning interval and circular-arc graphs into proper interval subgraphs with applications, in: LATIN 2004, LNCS 2976, 2004.

13. M. C. Golumbic, Algorithmic Graph Theory and Perfect Graphs, Academic Press, New York, 1980.

14. T. F. Havel, The combinatorial distance geometry approach to the calculation of molecular conformation, Ph.D. thesis, University of California, Berkeley (1982).

15. H. Imai, T. Asano, Finding the connected component and a maximum clique of an intersection graph of rectangles in the plane, Journal of algorithms 4 (1983) 310-323.

16. A. Kostochka, Coloring intersection graphs of geometric figures with a given clique number, Contemporary mathematics 342 (2004) 127-138.

17. J. Kratochvil, A special planar satisfiability problem and a consequence of its NP-completeness, Disc. Appl. Math. 52 (1994) 233-252.

18. N. V. R. Mahadev, U. N. Peled, Threshold graphs and related topics, vol. 56, Elsevier, Amsterdam, 1995.

19. T. S. Michael, T. Quint, Sphericity, cubicity, and edge clique covers of graphs, Disc. Appl. Math. 154 (8) (1984) 1309-1313.

20. F. S. Roberts, Recent Progresses in Combinatorics, chap. On the boxicity and Cubicity of a graph, Academic Press, New York, 1969, pp. 301-310.

21. E. R. Scheinerman, Intersection classes and multiple intersection parameters, Ph.D. thesis, Princeton University (1984).

22. C. Thomassen, Interval representations of planar graphs, J. Combin. Theory Ser. B 40 (1986) 9-20.

23. W. T. Trotter, Jr., A forbidden subgraph characterization of Roberts' inequality for boxicity, Disc. Math. 28 (1979) 303-314.

24. M. Yannakakis, The complexity of the partial order dimension problem, SIAM J. Alg. Disc. Math. 3 (3) (1982) 351-358. 